\title{
Antibiofilm Activity of Selected Plant Essential Oils and their Major Components
}

\author{
ALEKSANDRA BUDZYŃSKA ${ }^{1}$, MARZENA WIĘCKOWSKA-SZAKIEL ${ }^{1}$, BEATA SADOWSKA ${ }^{1}$, \\ DANUTA KALEMBA ${ }^{2}$ and BARBARA RÓŻALSKA ${ }^{{ }^{*}}$ \\ ${ }^{1}$ Institute of Microbiology, Biotechnology and Immunology, University of Łódź, Poland \\ ${ }^{2}$ Institute of General Food Chemistry, Technical University of Łódź, Poland
}

Received 14 October 2010, accepted 15 December 2010

\begin{abstract}
The aim of the study was to examine the antibiofilm activity of selected essential oils (EO): Lavandula angustifolia (LEO), Melaleuca alternifolia (TTO), Melissa officinalis (MEO) and some of their major constituents: linalool, linalyl acetate, $\alpha$-terpineol, terpinen-4-ol. Biofilms were formed by Staphylococcus aureus ATCC 29213 and Escherichia coli NCTC 8196 on the surface of medical biomaterials (urinary catheter, infusion tube and surgical mesh). TTC reduction assay was used for the evaluation of mature biofilm eradication from these surfaces. Moreover, time-dependent eradication of biofilms preformed in polystyrene 96-well culture microplates was examined and expressed as minimal biofilm eradication concentration (evaluated by MTT reduction assay). TTO, $\alpha$-terpineol and terpinen-4-ol as well as MEO, showed stronger anti-biofilm activity than LEO and linalool or linalyl acetate. Among the biomaterials tested, surgical mesh was the surface most prone to persistent colonization since biofilms formed on it, both by $S$. aureus and $E$. coli, were difficult to destroy. The killing rate studies of $S$. aureus biofilm treated with TTO, LEO, MEO and some of their constituents revealed that partial (50\%) destruction of 24-h-old biofilms $\left(\mathrm{MBEC}_{50}\right.$ ) was achieved by the concentration $4-8 \times \mathrm{MIC}$ after $1 \mathrm{~h}$, whereas $2-4 \times \mathrm{MIC}$ was enough to obtain $90 \%$ reduction in biomass metabolic activity $\left(\mathrm{MBEC}_{90}\right.$ ) after just $4 \mathrm{~h}$ of treatment. A similar dose-dependent effect was observed for $E$. coli biofilm which, however, was more susceptible to the action of phytochemicals than the biofilms of $S$. aureus. It is noteworthy that an evident decrease in biofilm cells metabolic activity does not always lead to their total destruction and eradication.
\end{abstract}

K e y words: biofilm eradication, biomaterial-associated infections, essential oils

\section{Introduction}

A frequent complication while using artificial devices in medical practice (BAI, biomaterial-associated infections) are infections dependent on microbial adhesion and biofilm formation. They should be considered a serious health problem requiring a rapid solution. Very often BAI are a consequence of direct biomaterial contamination during implantation but they can also be caused by haematogenous spread of bacteria from an infection site anywhere within the human body. A similar medical problem is posed by wound-associated chronic infections, which are also often characterized as having a biofilm nature. The biofilm mode of growth results in an increased bacterial resistance against classical antimicrobial treatment and host immune factors (Bryers, 2008; James et al., 2008; Dai et al., 2010; Hoiby et al., 2010).
Thus, the search for alternative therapies is a necessity and using, for example, natural plant/animal products and/or their combinations with antibiotics or synthetic counterparts seems to be one of the promising solutions (Dürig et al., 2010).

Higher plants evolved through developing mechanisms for avoiding the effects of microbial attack based on the production of protective substances, usually their secondary metabolites. Compounds with strong bacteriostatic or bactericidal activity belong mostly to phytoalexins and, within this group, essential oils are the most important members (Gibbons, 2008). Essential oils are complex mixtures of chemical substances, at least one of which shows antimicrobial activity. They consist mainly of monoterpenes, sesquiterpenes, diterpenes and other aromatic or aliphatic compounds (Kalemba and Kunicka, 2003; Bakkali et al., 2008; Reichling et al., 2009). Essential oils of several plants

* Corresponding author: B. Różalska, Department of Immunology and Infectious Biology, Institute of Microbiology, Biotechnology and Immunology, University of Łódź; Banacha 12/16, 90-237 Łódź, Poland; e-mail: rozab@biol.uni.lodz.pl 
are widely used in ethnomedicine for their antimicrobial and anti-inflammatory properties but their antibiofilm activity has not been so far studied extensively (Carson et al., 2006; Fabian et al., 2006; Cavanagh and Wilkinson, 2002; Karpanen et al., 2008; Warnke et al., 2006; Chao et al., 2008; Gursoy et al., 2009; Hossainzadegan and Delfan, 2009).

Essential oils derived from Melaleuca alternifolia, Lavandula angustifolia, Melissa officinalis and their major constituents were selected for the present study. Tea tree oil (TTO) is an essential oil from the leaves of the Australian M. alternifolia tree, a member of the botanical family Myrtaceae. The oil from the leaves is used medicinally, being effective against bacterial, viral and fungal organisms as well as having immunostimulatory activity. It is also known that it can alleviate inflammation and may help wound healing (Carson et al., 2006). Essential oils distilled from members of the genus Lavandula have been applied both cosmetically and therapeutically for centuries with the most commonly used species being $L$. angustifolia, L. latifolia, L. stoechas and L. intermedia. The claims made for lavender oil include its antibacterial, antifungal, smooth muscle relaxing, sedative, antidepressive properties (Cavanagh and Wilkinson, 2002; Roller et al., 2009). Melissa is commonly used in Europe as a tea, liquid extract, and topical preparation. Melissa essential oil (lemon balm) is also a common antibacterial and antifungal agent (Mimica-Dukic et al., 2004).

The attention of our study was focused on the possibility of using essential oils in the fight against biofilms of two members of the "alert" pathogens group. These were Gram-positive staphylococci, Staphylococcus aureus and Gram-negative enteric bacteria - Escherichia coli, since the epidemiological data on the incidence of infection with their participation sound serious. Moreover, these strains were chosen because the known differences in the cell wall structures between Gram-positive and Gram-negative bacteria imply the extent of their susceptibility to various antimicrobial agents (Gibbons, 2008; Fabian et al., 2006; Chao et al., 2008).

\section{Experimental}

\section{Materials and Methods}

Biomaterials, bacteria, phytochemicals. The following biomaterials/culture surfaces were used: short term urine drainage catheter of Nelaton type (Bicakcilar, Turkey), infusion tube (Polfa Lublin, Poland) (both $0.5 \mathrm{~cm}$ in length); surgical mesh support for muscle/fascia (Tricomed, Łódź, Poland) $(0.5 \times 0.5 \mathrm{~cm})$; 96 -well polystyrene tissue culture microplates (Nunc, Denmark). Eradication of Staphylococ- cus aureus ATCC 29213 and Escherichia coli NCTC 8196 biofilms by essential oils (EO) and some of their main components was evaluated. There were oils of Melaleuca alternifolia (tea tree oil, TTO), Lavandula angustifolia (lavender essential oil, LEO), Melissa officinalis (Melissa essential oil, MEO or lemon balm) and $\alpha$-terpineol, terpinen-4-ol, linalool, linalyl acetate. All essential oils were purchased from Pollena Aroma, Poland and the isolated compounds of EO were purchased from SAFC, USA, via Sigma, USA.

Evaluation of bacterial susceptibility to essential oils and their major constituents. MIC (minimal inhibitory concentration) of phytochemicals was determined by the standard CLSI (Clinical Laboratory Standards Institute) microdilution method, with minor modifications (Budzyńska et al., 2009). Essential oils/ components were initially diluted in $96 \%$ ethanol $(1: 1 \mathrm{v} / \mathrm{v})$, and later in Mueller-Hinton Broth (MHB, Sigma) with $0.5 \%$ Tween 20 (Sigma). The tested concentrations of phytochemicals (range $6.75-0.024 \%$ ) were deposited in triplicate, in a volume of $100 \mu \mathrm{l}$ in the wells of flat-bottomed polystyrene 96-well microplates (Nunc, Denmark). Then, $100 \mu 1$ of bacterial suspension with a density of $10^{6} / \mathrm{ml}$ in $\mathrm{MHB} / 0.5 \%$ Tween 20 was added to each well. The positive control was a suspension of bacteria in $200 \mu 1$ of $\mathrm{MHB} / 0.5 \%$ Tween 20, and a negative control was the medium without bacteria. After $24 \mathrm{~h}$ incubation at $37^{\circ} \mathrm{C}$, the absorbance at $\mathrm{A}_{600}$ (Victor 2, Wallac, Finland) was determined. The concentration at which the $\mathrm{A}_{600}$ of the wells did not exceed the value of absorbance of the control medium was accepted as the MIC. The lowest concentration of essential oils/components, bactericidal to $\geq 99.9 \%$ of the original inoculum (MBC, minimal bactericidal concentration) was determined from broth dilution MIC tests, by subculturing $100 \mu \mathrm{l}$ (from the wells of MIC, $2 \times$ MIC, $4 \times$ MIC, $8 \times$ MIC) to the broth medium without any antimicrobial agents. No visible growth after subsequent $24 \mathrm{~h}$ incubation means MBC on condition that the concentration is not higher than the 4-fold value of MIC. Antimicrobial substances - oxacillin and ofloxacin (Sigma, USA) were accepted as a reference.

Surface colonization and biofilm eradication. Fragments of biomaterials (three pieces of each type) were incubated with bacterial suspension in tryptic soy broth (TSB) supplemented with glucose (TSB/0.25\% Glc) for $24 \mathrm{~h}$ at $37^{\circ} \mathrm{C}$. Colonized surfaces, after their washing with phosphate-buffered saline (PBS), were transferred to the wells of a 96-well tissue culture microplate containing dilutions of essential oils/components and were incubated for the next $24 \mathrm{~h}$ at $37^{\circ} \mathrm{C}$. Then, biomaterial fragments were rinsed with PBS and transferred to a new 96-well microplate containing TSB with $1 \%$ TTC (2,3,5-triphenyltetrazolium chloride) for $24 \mathrm{~h}$ incubation at $37^{\circ} \mathrm{C}$, as previously 
described (Różalska et al., 1998). TTC is a redox indicator used to differentiate between metabolically active and inactive cells; the colorless compound is enzymatically reduced to red TPF (1,3,5-triphenylformazan) in living cells due to the activity of various dehydrogenases. The presence/reduction of biofilm biomass was evaluated by comparing the color intensity of biofilm deposit on biomaterials treated and untreated with phytochemicals, according to arbitrarily fixed scale $(4+, 3+, 2+, 1+,-)$. The experiments were performed twice to confirm reproducibility of the results.

The results of the semi-quantitative method were compared with those of a standard biomass quantification method by CFU determination. The fragments of colonized biomaterials treated with phytochemicals, which were scored by TTC assay as (+) and (-), were removed from the wells and rinsed in PBS. Then, they were placed in $1 \mathrm{ml}$ of sterile broth medium and the remaining bacterial deposit was removed by ultrasonic disruption for $5 \mathrm{~min}$. One hundred $\mu \mathrm{l}$ of the sonicate was spread onto two agar plates for overnight incubation and CFU counting.

Time-dependent biofilm eradication. Time-dependent inhibitory concentration of phytochemicals for biofilms preformed in a polystyrene 96-well culture microplate, used at concentrations from MIC to $8 \times$ MIC was determined. Mature (24-h old) biofilms of $S$. aureus ATCC 29213 and E. coli NCTC 8196 were exposed to the action of essential oils/components for $1,4,6$ and $24 \mathrm{~h}$ co-incubation at $37^{\circ} \mathrm{C}$. The essential oils/components concentrations causing $50 \%$ or at least $90 \%$ reduction in biomass metabolic activity $\left(\mathrm{MBEC}_{50}\right.$ and $\left.\mathrm{MBEC}_{90}\right)$ at each time point were determined using MTT-reduction assay, as described earlier (Walencka et al., 2005; 2007). $\mathrm{MBEC}_{50}$ means that $\mathrm{A}_{550}$ of the sample dropped below the half value of the positive control $\left(\mathrm{A}_{550}=1.5\right)$ and $\mathrm{MBEC}_{90}$ means that $A_{550}$ of the sample was close to the value of the negative control $\left(\mathrm{A}_{550}=0.2\right)$. To avoid interference between the phytochemical's red-ox potency and MTT, before its application, the fluid above the biofilm was aspirated and removed. In this assay, pale-yellow MTT (3-(4,5-dimethylthiazol-2-yl)-2,5-diphenyltetrazolium bromide) is reduced to purple formazan by living, respiratory active cells. A solubilizer was added to dissolve the formed formazan product into a colored solution whose absorbance was quantified $(550 \mathrm{~nm}$ (Victor 2, Wallac, Finland). The experiments were performed in duplicate to confirm reproducibility of the results. Mean $\mathrm{A}_{550} \pm \mathrm{S}$.D. values were presented.

Evaluation of viability of bacterial cells treated with essential oils. The potential effect of essential oil on bacterial cell membranes was assessed using EO-mediated SYTO 9 and propidium iodide uptake (LIVE/DEAD BacLight Bacterial Viability kit, Molecular Probes, Invitrogen, Poland). Cell suspensions of
S. aureus ATCC 29213 and E. coli NCTC 8196 in PBS $\left(1.0 \times 10^{6} \mathrm{CFU} / \mathrm{ml}\right)$ were incubated with $4 \times \mathrm{MIC}$, $2 \times \mathrm{MIC}$ or MIC) of $\mathrm{EO}$ at $37^{\circ} \mathrm{C}$ for $4 \mathrm{~h}$, with shaking. After the incubation, the bacteria were washed with $5 \mathrm{ml}$ sterile PBS and stained using the LIVE/DEAD kit, according to the manufacturer's recommendations. The cells with LIVE/DEAD and no EO served as negative controls. After the incubation all the samples were washed and resuspended in PBS, and a drop of each suspension was examined with a confocal scanning laser microscope for green/red fluorescence to visualize SYTO 9 and propidium iodide, respectively. Six areas of each of the triplicate samples were photographed with an integrated Hamamatsu digital camera (C4742-95; Nikon UK).

\section{Results}

The minimal inhibitory concentration (MIC) of selected essential oils and their major constituents was determined using the broth microdilution method and defined as the lowest concentration able to inhibit visible microbial growth. The essential oil of Melaleuca alternifolia (TTO), $\alpha$-terpineol and terpinen-4-ol, as well as the essential oil of Melissa officinalis (MEO), showed stronger antibacterial activity than the essential oil of Lavandula angustifolia (LEO) and its major components, such as linalool and linalyl acetate. Their MICs values against Staphylococcus aureus and Escherichia coli are presented in Table I. The experiments performed to check whether the tested phytochemicals are bactericidal showed that MBC values did not exceed the obligatory $4 \mathrm{x}$ MIC, since more than $99 \%$ of the bacterial inoculum was killed even by MIC or $2 \times$ MIC. One exception was the $S$. aureus culture which was killed by $8 \times$ MIC of linalool - one of the components from the lavender and melissa essential oils. The data on this set of experiments are presented in Table I.

The answer to the question whether TTO, LEO, MEO are bacteriostatic or bactericidal was also provided by the experiments for EO-mediated SYTO 9/PI uptake, evaluated by fluorescent confocal microscopy. As assessed by the emission of green/red fluorescence, non-treated $S$. aureus or E. coli cells, showed only green fluorescence (live with undamaged cell wall), except for very few red cells. However, EO-treated cells exhibited increased uptake of propidium iodide (leaking cell wall and membrane), proportional to the increase in the compound concentration. All bacterial cells from planktonic cultures treated with EO at the concentration of 2-4 MIC, exhibited red fluorescence (data not shown).

In the further experiments, essential oils/compounds were shown to possess the bactericidal activity against 


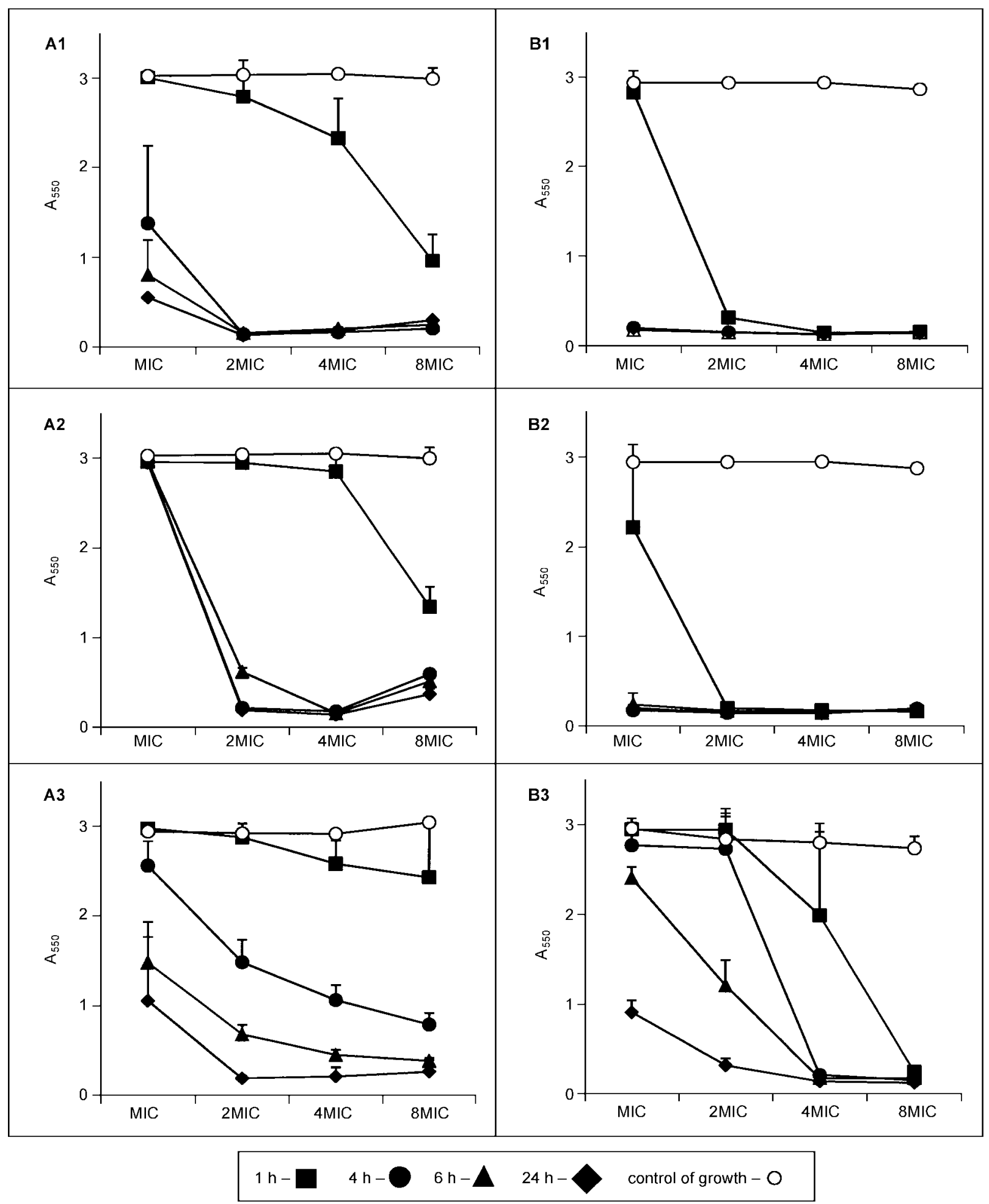

Fig. 1. Time- and concentration-dependent effect of essential oils on biofilm viability, determined by MTT-reduction assay. A1, A2, A3 (S. aureus), B1, B2, B3 (E. coli) biofilms exposed to tea tree oil (A1, B1), lavender oil (A2, B2), or lemon balm (A3, B3). $\mathrm{A}_{550}$ mean \pm S.D.

mature S. aureus and E. coli biofilms formed earlier on the surface of polystyrene culture microplate wells. The time-dependent inhibitory concentration of phytochemicals for biofilms preformed in the polystyrene 96-well culture microplate was determined by MTT reduction assay. All biocides were used for this pur- pose at MIC up to $8 \times$ MIC. There was only a small difference between planktonic and biofilm biocidal concentrations, usually not exceeding 2-4-fold higher values. Killing rate studies of $S$. aureus biofilm eradication by TTO and its constituents revealed that partial $(50 \%)$ destruction of 24-h-old biofilms $\left(\mathrm{MBEC}_{50}\right)$ 
Table I

Bacteriostatic and bactericidal activity of essential oils/consituents against planktonic and biofilm cultures of Staphylococcus aureus and Escherichia coli

\begin{tabular}{|l|l|c|c|c|c|}
\hline $\begin{array}{c}\text { Essential } \\
\text { oil/component }\end{array}$ & Bacteria & $\begin{array}{c}\text { MIC (planktonic), } \\
\% \mathrm{v} / \mathrm{v}\end{array}$ & $\begin{array}{c}\text { MBC (planktonic) } \\
\% \mathrm{v} / \mathrm{v}\end{array}$ & $\begin{array}{c}\text { MBEC }_{90} \text { (4 hours), } \\
\% \mathrm{v} / \mathrm{v}\end{array}$ & $\begin{array}{c}\text { MBEC }_{90} \text { (24 hours), } \\
\% \mathrm{v} / \mathrm{v}\end{array}$ \\
\hline \multirow{2}{*}{ TTO } & S. aureus & 0.39 & 0.78 & 0.78 & 0.78 \\
\cline { 2 - 6 } & E. coli & 0.19 & 0.19 & 0.19 & 0.19 \\
\hline \multirow{2}{*}{$\alpha$-terpineol } & S. aureus & 0.19 & 0.78 & 0.38 & 0.38 \\
\cline { 2 - 6 } & E. coli & 0.097 & 0.097 & 0.19 & 0.19 \\
\hline \multirow{2}{*}{ Terpinen-4-ol } & S. aureus & 0.19 & 0.78 & 0.19 & 0.19 \\
\cline { 2 - 6 } & E. coli & 0.048 & 0.19 & 0.097 & 0.048 \\
\hline \multirow{2}{*}{ LEO } & S. aureus & 0.78 & $1.56^{*}$ & 1.56 & 1.56 \\
\cline { 2 - 6 } & E. coli & 0.19 & 0.19 & 0.19 & 0.19 \\
\hline \multirow{2}{*}{ Linalool } & S. aureus & 0.19 & 1.56 & 0.78 & 0.78 \\
\cline { 2 - 6 } & E. coli & 0.097 & 0.39 & 0.78 & 0.19 \\
\hline \multirow{2}{*}{ Linalyl acet. } & S. aureus & 0.19 & 0.78 & $>1.56$ & 0.19 \\
\cline { 2 - 6 } & E. coli & 0.19 & 0.19 & $>1.56$ & 0.19 \\
\hline \multirow{2}{*}{ MEO } & S. aureus & 0.097 & 0.19 & 0.78 & 0.38 \\
\cline { 2 - 6 } & E. coli & 0.048 & 0.097 & 0.19 & 0.19 \\
\hline
\end{tabular}

TTO - Tea Tree oil (Melaleuca alternifolia); LEO - lavender oil (Lavandula angustifolia)

MEO - Lemon balm oil (Melissa officinalis); MIC - minimal inhibitory concentration

$\mathrm{MBC}-$ minimal bactericidal concentration, value not exceded $4 \times$ MIC;

* - exception linalool which is not bactericidal for $S$. aureus, $\mathrm{MBC}=8 \times \mathrm{MIC}$

MBEC $_{90}$ - minimal biofilm eradication concentration causing $\geq 90 \%$ reduction of biomass

metabolic activity, measured after 4 or $24 \mathrm{~h}$ of compounds application on the biofilm culture

was achieved by the concentration of $4-8 \times$ MIC just after $1 \mathrm{~h}$, whereas $2 \times \mathrm{MIC}$ was enough to obtain TTO $\mathrm{MBEC}_{90}$ after $4 \mathrm{~h}$ of treatment. Effective $\mathrm{MBEC}_{90}$ of LEO and its constituents was as high as $4-8 \times$ MIC. The time-dependent activity of MEO was comparable to TTO. With few exceptions, the tested phytochemicals reduced the metabolic activity of bacterial cells in biofilms after $24 \mathrm{~h}$ exposure at concentrations close to MICs. A similar dose- and time-dependent effect was observed for $E$. coli biofilm, however, surprisingly, it was more susceptible to the action of phytochemicals than the $S$. aureus biofilms, similarly to what was observed for planktonic cultures. (Table I, Fig. 1).

Among the medical biomaterials tested, surgical mesh was the surface most prone to persistent colonization, since biofilms formed on it by both bacterial strains were more difficult to eradicate by the tested compounds. Modified Richard's method was used to detect the presence or eradication of biofilm, as described earlier (Różalska et al., 1998). The obtained visual results scored by TTC assay as (+) and (-), were compared with the data from the standard biomass quantification method by bacterial dislodgement and CFU determination. Only TTO and terpinen-4-ol used at MIC $-2 \times$ MIC caused visible biofilm eradication (TTC reduction) from the surface of urological catheter and infusion tube, evaluated later as greater than $90 \%$ decrease in the number of live bacteria (CFU). However, the use of higher concentrations $(4-8 \times$ MIC) of these and other compounds (MEO,
LEO, $\alpha$-terpineol, linalool, linalyl acetate) was necessary to achieve such an effect on the surface of surgical mesh. In all cases it was proved that indeed TTC score $(-)$ means that the lack of metabolically active cells is equal to the lack of viable bacteria, since the CFU counting method used for the measurement of biofilm survival showed its total eradication (data not shown).

Our study leads to the conclusion that essential oils and some of their major constituents are able to efficiently reduce biofilms of both $S$. aureus and $E$. coli on biomaterial surfaces. However, a satisfactory effect is strongly dependent on the surface structure and properties. The example of TTO antibiofilm activity is presented in Fig. 2.

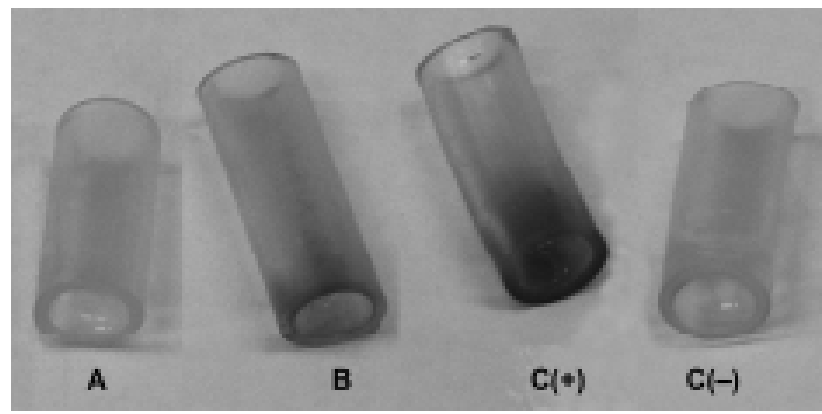

Fig. 2. Image of infusion tube colonized by $S$. aureus biofilm: A, B - treated for $24 \mathrm{~h}$ with TTO, respectively at MIC or $1 / 2 \mathrm{MIC}$. $\mathrm{C}(+)$ - not treated positive control, $\mathrm{C}(-)$ - not colonized negative control. TTC-reduction assay - scored according to an arbitrarily fixed scale as described in Material and Methods. 


\section{Discussion}

In the present study, the in vitro strong effects of Melaleuca alternifolia (TTO), Lavandula angustifolia (LEO), Melissa officinalis (MEO) essential oils and some of their main constituents ( $\alpha$-terpineol, terpinen4-ol, linalool, linalyl acetate) on the biofilms of Grampositive $S$. aureus and Gram-negative E. coli were shown. Remarkably, the in vitro activity of the oils against the biofilm of both strains tested was equal to or only slightly lower than that against bacterial planktonic culture (Table I). This is worth emphasizing because the biofilm resistance to classical chemotherapeutics is typically 100-1000 times higher (Bryers, 2008, Hoiby et al., 2010). It is a promising observation since plant-derived compounds have gained a general interest in the search to identify alternatives for the control of infections, especially those connected with the use of artificial medical devices. Research on this topic is focused on natural or synthetic substances, which have potent broad-spectrum microbicidal and antibiofilm activities and pose a low risk for the development of resistance. It is accepted that there are two main reasons why essential oils do not create resistant strains of bacteria: they are complex and comprise numerous compounds in variable proportions depending on the plant chemotype. Hence, even if bacteria did figure out an oil's effective components, they would have to start all over with the next set (Bakkali et al., 2008; Reichling et al., 2009).

In the present study we demonstrated that essential oils, not only of M. alternifolia well known in this respect from other studies (Brady et al., 2006; Kwieciński et al., 2009; Karpanen et al., 2008), but also of M. officinalis and L. angustifolia have antibiofilm potency. According to our knowledge, this is the first report concerning such activity of MEO and LEO. Both $S$. aureus and E. coli biofilms were eradicated efficiently by MEO. However, this effect was more time-dependent than the activity of TTO and LEO. The antibacterial, antifungal and antioxidant properties of M. officinalis essential oil, but not antibiofilm activity, have been earlier described. The main constituents of MEO are citrals (geranial + neral, $39.9 \%)$, citronellal (13.7\%), limonene (2.2\%), geraniol (3.4\%), $\beta$-caryophyllene (4.6\%), $\beta$-caryophyllene oxide $(1.7 \%)$, and germacrene D $(2.4 \%)$ (Mimica-Dukic et al., 2004). Our further research will be devoted to examining the activity of individual components. However, it is also possible that they can be more active when they are in their natural proportions in the native oil. The main chemical components of lavender oil - the second active in our experiments are a-pinene, limonene, 1,8-cineole, cis-ocimene, transocimene, 3-octanone, camphor, linalool, linalyl acetate, caryophyllene, terpinen-4-ol and lavendulyl acetate
(Roller et al., 2009; Cavanagh and Wilkinson, 2002). Three of these components linalool, linalyl acetate, terpinen-4-ol were tested for antibiofilm activity since they are also major constituents of active TTO. Among them, terpinen-4-ol seems to have the most potent activity, causing more than $90 \%$ reduction in biofilm metabolic activity $\left(\mathrm{MBEC}_{90}\right)$ after just $4 \mathrm{~h}$ of exposure.

Our results on TTO antibiofilm activity are slightly different from those published by Brady et al. (2006). The authors showed that biofilms formed by MSSA and MRSA isolates were completely eradicated following an exposure to 5\% TTO for $1 \mathrm{~h}$. In our study, $S$. aureus biofilm was also eradicated in such a short time but only by $66 \%$. However, the concentration of TTO used was much lower $-3.1 \%(8 \times \mathrm{MIC})$. On the other hand, total eradication of $S$. aureus biofilm was achieved not earlier than after $4 \mathrm{~h}$ but the concentration of TTO needed for that was only $0.78 \%(2 \times \mathrm{MIC})$. Similarly to our results, TTO activity against $S$. aureus biofilms was reported by Kwieciński et al. (2009).

Usually plant-derived compounds show considerable activity against Gram-positive bacteria but not against Gram-negative species or yeast, which have evolved significant permeability barriers (Bakkali et al., 2008; Gibbons, 2008; Reichling et al., 2009; Amalradjou et al., 2010). Thus, it is worth emphasizing that in our experiments the Gram-negative bacteria were very susceptible to damage by all the essential oils used. For example, the potent and very fast antibiofilm activity of TTO was noticed for E. coli which was eradicated within $1 \mathrm{~h}$ exposure to concentration $0.78 \%$.

It is noteworthy that the evident decrease in biofilm cells metabolic activity measured by MTT-reduction assay (biofilms in microplate wells), is not equal to their total destruction and eradication from the surface of the real medical biomaterials. We, like some other authors, have defined a drug concentration resulting in $\geq 90$ biomass reduction measured by MTT staining as the MBEC (minimal biofilm eradication concentration). This method indeed has shown excellent applicability as it is able to detect dose-dependent and time-dependent differences in the effect of antimicrobials. But, contrary to other investigators, we have not found a strong correlation between the amount of biofilm mass exposed to essential oils, quantified with TTC staining on medical biomaterials and the metabolic activity quantified with MTT in the wells of microplate. A great advantage of the TTC reduction method is the fact that the metabolic reduction of colorless TTC into red-colored formazan by bacteria adhering to the surface of a synthetic implant occurs regardless of the type, shape or color of the biomaterial. However, this method is only semi-quantitative and requires confirmation using a more objective evaluation method, which was done in our study. This 
suggests that research on given compound's antibiofilm activity should be conducted using simultaneously at least two independent methods. It is well known that the growth conditions and the type of surface may affect the architecture of a biofilm which can influence the cells sensitivity to antimicrobial agents (Bryers, 2008; Flemming et al., 2009; Hoiby et al., 2010). However, our results encourage the exploration of the other essential oils and their constituents showing the most potent antibiofilm activity since the oils used in this study have superior antimicrobial activity in $S$. aureus and $E$. coli biofilms, compared with conventional antibiotics.

\section{Literature}

Amalaradjou M.A.R., A. Narayanan, S.A. Baskaran and K. Venkitanarayanan. 2010. Antibiofilm effect of trans-cinnamaldehyde on uropathogenic Escherichia coli. J. Urology. 184: 358-363.

Bakkali F., S. Averbeck, D. Averbeck and M. Idaomar. 2008. Biological efects of essential oils - A review. Food Chem. Toxicol. 46: 446-475.

Brady A., R. Loughlin, D. Gilpin, P. Paddy Kearney and M. Tunney. 2006. In vitro activity of tea-tree oil against clinical skin isolates of meticillin-resistant and -sensitive Staphylococcus aureus and coagulase-negative staphylococci growing planktonically and as biofilms. J. Med. Microbiol. 55: 1375-1380.

Bryers J.D. 2008. Medical biofilms. Biotechnol. Bioeng. 100: 1-17. Budzyńska A., M. Więckowska-Szakiel, D. Kalemba, B. Sadowska and B. Różalska. 2009. The optimization of methods utilized for testing the antibacterial activity of essential oils (in Polish). Med. Dośw. Mikrobiol. 61: 281-281.

Carson C.F., K.A. Hammer and T.V. Riley. 2006. Melaleuca alternifolia (Tea Tree) oil: a review of antimicrobial and other medicinal properties. Clin. Microbiol. Rev. 19: 50-62.

Cavanagh H.M.A. and J.M. Wilkinson. 2002. Biological activities of lavender essential oil. Phytotherapy Res. 16: 301-308.

Chao S., G. Young, C. Oberg and K. Nakaoka. 2008. Inhibition of methicillin-resistant Staphylococus aureus (MRSA) by essential oils. Flavour and Fragrance J. 23: 444-449.

Dai T., Y-Y. Huang, K.S. Sharma, T.J. Hashmi, B.D. Kurup and R.M. Hamblin. 2010. Topical antimicrobials for burn wound infections. Recent Patents on Anti-Infective Drug Discovery 5: 124-151.

Dürig A., I. Kouskoumvekaki, R.M. Vejborg and P. Klemm. 2010. Chemoinformatics-assisted development of new antibiofilm compounds. Appl. Microbiol. Biotechnol. 87: 309-317.

Fabian D., M. Sabol, K. Domaracka and D. Bujnakova. 2006. Essential oils - their antimicrobial activity against Escherichia coli and effect on intestinal cell viability. Toxicology in vitro 20 : $1435-1445$.
Flemming K., C. Klingenberg, J.P. Cavanagh, M. Sletteng, W. Stensen, J.S. Svendsen and T. Flćgstad. 2009. High in vitro antimicrobial activity of synthetic antimicrobial peptidomimetics against staphylococcal biofilms. J. Antimicrob. Chemother. 63: 136-145.

Gibbons S. 2008. Phytochemicals for bacterial resistance - strengths, weaknesses and opportunities. Planta Med. 74: 594-602.

Gursoy U.K., M. Gursoy, O.V. Gursoy, L. Cakmakci, E. Kononen and V-J. Uitt. 2009. Antibiofilm properties of Satureja hortensis L. essential oil against periodontal pathogens. Anaerobe, 15: 164-167.

Hoiby N., T. Bjarnsholt, M. Givskov, S. Molin and O. Ciofu. 2010. Antibiotic resistance of bacterial biofilm. Int. J. Antimicrob. Agents 35: 322-332.

Hossainzadegan H. and Delfan B. 2009. Evaluation of antibiofilm activity of dentol. Acta Med. Iranica. 47: 35-40.

James G.A., E. Swogger, R. Wolcott, E. Pulcini, P. Secor, J. Sestrich, J.W. Costerton and P.S. Stewart. 2008. Biofilms in chronic wounds. Wound Repair Regen. 16: 37-44.

Kalemba D. and A. Kunicka. 2003. Antibacterial and antifungal properties of essential oils. Curr. Med. Chem. 10: 813-29.

Karpanen T.J., T. Worthington, E.R. Hendry, B.R. Conway and P.A. Lambert. 2008. Antimicrobial efficacy of chlorhexidine digluconate alone and in combination with eucalyptus oil, tea tree oil and thymol against planktonic and biofilm cultures of Staphylococcus epidermidis. J. Antimicrob. Chemother. 62, 1031-1036.

Kwieciński J., S. Eick and K. Wójcik. 2009. Effects of tea tree (Melaleuca alternifolia) oil on Staphylococcus aureus in biofilms and stationary growth phase. Int. J. Antimicrob. 33: 343-347.

Mimica-Dukic N., B. Bozin, M. Sokovic, and N. Simin. 2004. Antimicrobial and antioxidant activities of Melissa officinalis L. (Lamiaceae) essential oil. J. Agric. Food Chem. 52: 2485-2489.

Reichling J., P. Schnitzler, U. Suschke and R. Saller. 2009. Essential oils of aromatic plants with antibacterial, antifungal, antiviral, and cytotoxic properties - an overview. Forsch Komplementmed. 16: 79-90.

Roller S., N. Ernest and J. Buckle. 2009. The antimicrobial activity of high-necrodane and other lavender oils on methicillin-sensitive and -resistant Staphylococcus aureus (MSSA and MRSA). J. Altern. Complement Med. 15: 275-279.

Różalska B., B. Sadowska, M. Więckowska and W. Rudnicka. 1998. Detection of biomaterial-associated bacterial biofilm (in Polish). Med. Dośw. Mikrobiol. 50: 115-122.

Walencka E., B. Sadowska, S. Różalska, W. Hryniewicz and B. Różalska. 2005. Lysostaphin as a potential therapeutic agent for staphylococcal biofilm eradication. Pol. J. Microbiol. 54: 191-200.

Walencka E., S. Różalska, H. Wysokińska, M. Różalski, L. Kuźma and B. Różalska. 2007. Salvipisone and aethiopinone from Salvia sclarea hairy roots modulate staphylococcal antibiotic resistance and express antibiofilm activity. Planta Med. 73: 545-551. Warnke P.H., E. Sherry, P.A.J. Russo, Y. Acil, J. Wiltfang, S. Sivananthan, M. Sprengel, J.C. Roldan, S. Schubert, J.P. Bredee and others. 2006. Antibacterial essential oils in malodorous cancer patients: Clinical observations in 30 patients. Phytomedicine 13: 463-467. 